\title{
Early responses of the STAT3 pathway to platinum drugs are associated with cisplatin resistance in epithelial ovarian cancer
}

\author{
W.J. Sheng ${ }^{1}$, H. Jiang ${ }^{1}$, D.L. $\mathrm{Wu}^{2}$ and J.H. Zheng ${ }^{1}$ \\ ${ }^{1}$ Department of Obstetrics and Gynecology, The First Affiliated Hospital of Harbin Medical University, Harbin, China \\ ${ }^{2}$ The Key Laboratory of Veterinary Public Health, Ministry of Agriculture, State Key Laboratory of Veterinary Biotechnology, \\ Harbin Veterinary Research Institute, Chinese Academy of Agriculture Sciences, Harbin, China
}

\begin{abstract}
Cisplatin resistance remains one of the major obstacles when treating epithelial ovarian cancer. Because oxaliplatin and nedaplatin are effective against cisplatin-resistant ovarian cancer in clinical trials and signal transducer and activator of transcription 3 (STAT3) is associated with cisplatin resistance, we investigated whether overcoming cisplatin resistance by oxaliplatin and nedaplatin was associated with the STAT3 pathway in ovarian cancer. Alamar blue, clonogenic, and wound healing assays, and Western blot analysis were used to compare the effects of platinum drugs in SKOV-3 cells. At an equitoxic dose, oxaliplatin and nedaplatin exhibited similar inhibitory effects on colony-forming ability and greater inhibition on cell motility than cisplatin in ovarian cancer. Early in the time course of drug administration, cisplatin increased the expression of pSTAT3 (Tyr705), STAT3 $\alpha$, VEGF, survivin, and Bcl-X $X_{L}$, while oxaliplatin and nedaplatin exhibited the opposite effects, and upregulated pSTAT3 (Ser727) and STAT3 $\beta$. The STAT3 pathway responded early to platinum drugs associated with cisplatin resistance in epithelial ovarian cancer and provided a rationale for new therapeutic strategies to reverse cisplatin resistance.
\end{abstract}

Key words: Epithelial ovarian cancer; Cisplatin; Oxaliplatin; Nedaplatin; STAT3

\section{Introduction}

Epithelial ovarian cancer accounts for approximately $90 \%$ of ovarian malignancies and is often discovered at an advanced stage, with a 5-year survival rate of less than $20 \%$ (1). While administration of cisplatin remains the current standard of care in chemotherapy for this malignancy, $75 \%$ of the patients eventually relapse (2). Cisplatin promotes development of drug resistance, thus there is an urgent need to overcome cisplatin resistance and identify its mechanisms.

Nedaplatin, a second-generation platinum drug, has a higher water solubility than cisplatin and causes fewer side effects (3). Oxaliplatin, a third-generation platinum compound, displays wide antitumor activity against cancers restricted to the peritoneal cavity and showed partial cross-resistance to cisplatin in preclinical studies (4). Both nedaplatin and oxaliplatin are effective in heavily pretreated ovarian cancer patients who are nonresponsive to cisplatin, with manageable toxicity (4-6). However, the mechanism underlying their reversal of cisplatin resistance remains largely unknown. Exposure to chemotherapeutic agents can induce alterations in signaling cascades that mediate resistance (7). Among these, signal transducer and activator of transcription 3 (STAT3) overexpression has been reported positively associated with cisplatin resistance (8).

STAT3 is activated in $94 \%$ of ovarian cancers and is associated with more aggressive phenotypes (7-9). It also correlates with recurrent tumors and poor prognosis (7-10). Furthermore, STAT3 participates in various processes key to malignant progression: promoting cell proliferation, triggering tumor initiation, migration, invasion, angiogenesis and metastasis, induction of epithelialmesenchymal transition (EMT), inhibition of apoptosis and cell cycle dysregulation, and promoting multidrug resistance to chemotherapy (8-13). STAT3 also plays an important role in embryonic stem cells and the immune

Correspondence: J.H. Zheng, 23 Youzheng Street, Nangang Block, Harbin, HLJ 150001, China. Fax: + 86-451-8268-1772. E-mail: zhengjhyd@yahoo.com.cn 
response $(14,15)$ partially correlated with cancer progression. Targeting STAT3 may reverse the malignant progression described above.

Since oxaliplatin and nedaplatin have been effective against cisplatin-resistant ovarian cancer in clinical trials, and targeting STAT3 may reverse drug resistance, we investigated whether the effects of platinum agents on the STAT3 pathway were associated with cisplatin resistance. We observed that oxaliplatin and nedaplatin had various effects on the STAT3 pathway that differed from that of cisplatin early in the course of treatment, which may contribute to elucidating their mechanisms of reversing cisplatin resistance.

\section{Material and Methods}

\section{Material}

Platinum drugs were from Aosaikang Pharmaceutical Co., Ltd. (Nanjing, China). Alamar blue was purchased from Invitrogen (USA). RPMI 1640, FBS, antibiotics, and trypsin were from Gibco (USA). Nitrocellulose membranes were from Amersham (UK). Primary antibodies were purchased from Santa Cruz Biotechnology (USA), except for PSTAT3 and Tyr705, which were from CST (USA). Secondary antibodies were from Dako (Denmark). Electrochemiluminescence (ECL) assay reagents were obtained from Pierce (USA). All other reagents and compounds were of analytical grade.

\section{Cell lines and cultures}

Human ovarian cancer SKOV-3 cells were from ATCC and cultured in RPMI 1640 medium with $10 \%$ FBS, $100 \mathrm{lU} / \mathrm{mL}$ penicillin, and $100 \mu \mathrm{g} / \mathrm{mL}$ streptomycin. Cells were cultured at $37^{\circ} \mathrm{C}$ in a humidified $5 \% \mathrm{CO}_{2}$ atmosphere.

\section{Proliferation assay}

SKOV-3 cells were seeded at $1 \times 10^{3}$ cells/well on 96-well plates. Drugs were added at the indicated doses ( $n=6$ for each experiment) $24 \mathrm{~h}$ after inoculation. The proliferation assay was performed 24,48 , and $72 \mathrm{~h}$ after drug treatment using the Alamar blue assay following the manufacturer's instructions. A microplate reader was used to measure the absorbance at 570 and $600 \mathrm{~nm}$. Absorbance recordings were normalized to control cells and graphed as relative cell density.

\section{Clonogenic assay}

At $24 \mathrm{~h}$ after exposure to $1 \mu \mathrm{g} / \mathrm{mL}$ cisplatin, $8 \mu \mathrm{g} / \mathrm{mL}$ oxaliplatin, or $8 \mu \mathrm{g} / \mathrm{mL}$ nedaplatin, SKOV-3 cells were seeded at 1000 cells/well on 6-well plates. Cells were cultured additionally for 10 days until visible colonies formed. The cells were fixed with methanol and stained with $0.5 \%$ crystal violet in $2 \%$ ethanol for $10 \mathrm{~min}$. The plates were washed 4 times with tap water and air-dried. Colonies comprising at least 50 cells were counted. The plating efficiencies were calculated as the number of colonies divided by the number of control cells plated.

\section{Wound healing assay}

Upon reaching $90 \%$ confluence on 6 -well plates, cells were scraped across the cell monolayer using a sterile 200- $\mu \mathrm{L}$ pipette tip. After washing with PBS, cells were exposed to $1 \mu \mathrm{g} / \mathrm{mL}$ cisplatin, $8 \mu \mathrm{g} / \mathrm{mL}$ oxaliplatin, or $8 \mu \mathrm{g} / \mathrm{mL}$ nedaplatin for $24 \mathrm{~h}$. Cells that migrated to the wounded region were photographed at $0 \mathrm{~h}$ and again after $24 \mathrm{~h}$. The wound area was quantified by Image $J$ and estimated using the following equation: wound closure $\%=[1-$ (wound area at $T_{t} /$ wound area at $\left.T_{0}\right) \times 100 \%$, where $T_{t}$ is the elapsed time after wounding and $T_{0}$ is the time immediately after wounding.

\section{Western blot analysis}

Cells $\left(1 \times 10^{6}\right)$ were seeded onto 6 -well plates and after treatment with $1 \mu \mathrm{g} / \mathrm{mL}$ cisplatin, $8 \mu \mathrm{g} / \mathrm{mL}$ oxaliplatin, or $8 \mu \mathrm{g} / \mathrm{mL}$ nedaplatin at the indicated time, proteins were extracted using RIPA buffer complemented with PMSF. The total protein concentration was determined by a BCA method. Equal amounts of total protein with $2 \times$ SDS were separated on $10 \%$ SDS-polyacrylamide electrophoresis gels and transferred to nitrocellulose membranes by semidry electrophoresis. The membranes were blocked in 5\% skim milk and incubated sequentially with the primary antibodies pSTAT3 (Tyr705), 1:1000; cyclin D1, 1:100; vascular endothelial growth factor (VEGF), 1:100; others, 1:200), followed by a secondary peroxidaseconjugated antibody (1:4000 dilution), and visualized with an ECL detection system. $\beta$-actin (1:500 dilution) was used as the control protein. The results were quantified by Image $J$ and were normalized to that of $\beta$-actin to obtain relative intensity.

\section{Statistical analysis}

All statistical analyses were performed using GraphPad Prism 5.0 (USA). Statistical significance was analyzed by one-way ANOVA and the Dunnett post hoc test, with values of $\mathrm{P}<0.05$ considered to be statistically significant. Data are reported as means \pm SE. Each experiment was repeated at least twice.

\section{Results}

\section{Equitoxic effects of platinum drugs in ovarian cancer cells}

We first evaluated the cytotoxic effects of cisplatin, oxaliplatin and nedaplatin in SKOV-3 cells, as their effects in ovarian cancer have not yet been compared. While cisplatin $(1 \mu \mathrm{g} / \mathrm{mL})$ inhibited cell proliferation by approximately $40-50 \%$ by $48 \mathrm{~h}$, oxaliplatin $(8 \mu \mathrm{g} / \mathrm{mL})$ and nedaplatin $(8 \mu \mathrm{g} / \mathrm{mL})$ demonstrated similar effects (Figure 1). These equitoxic concentrations were used in the subsequent experiments reported here in order to exclude cytotoxic effects. 
A

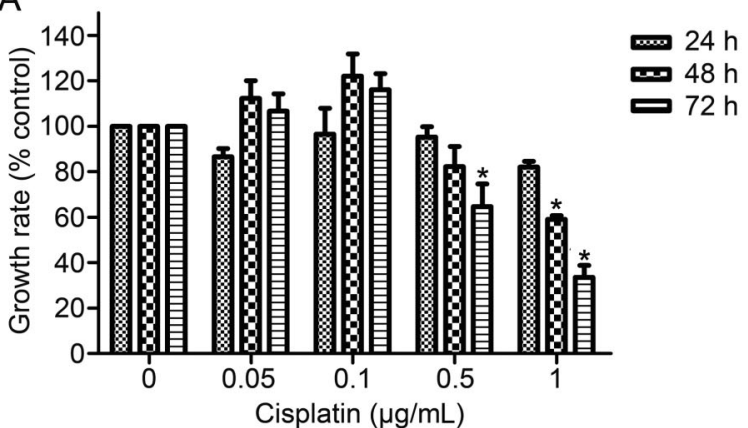

B

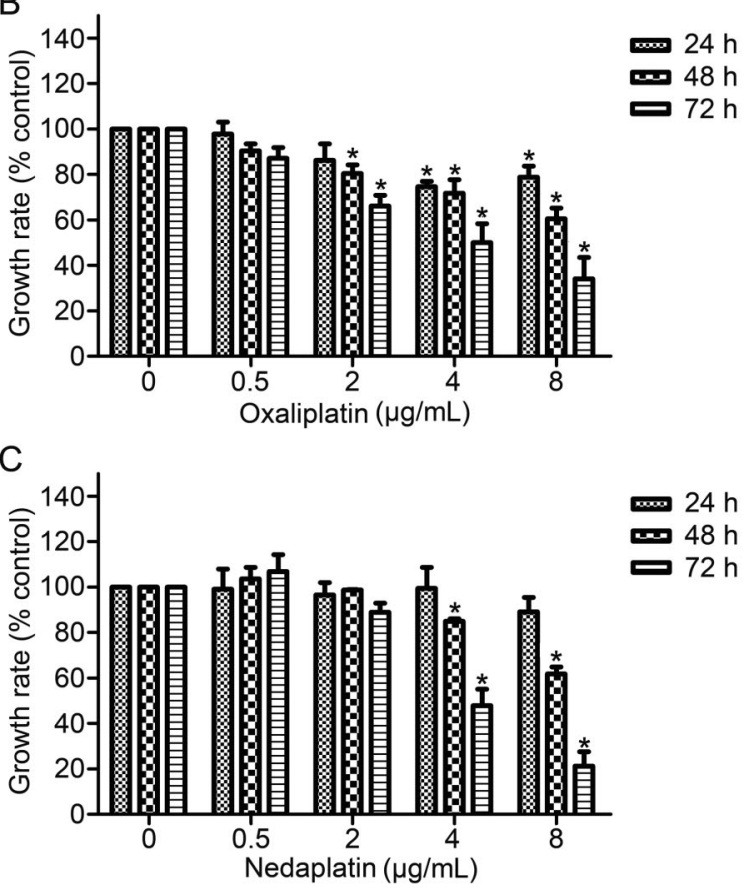

Figure 1. Effect of platinum drugs on proliferation of SKOV-3 cells. SKOV-3 cells $\left(1 \times 10^{3}\right)$ were seeded on 96-well plates in growth medium. After $24 \mathrm{~h}$ of inoculation, cells were stimulated with different concentrations of cisplatin $(A)$, oxaliplatin $(B)$, and nedaplatin $(C)$ for indicated times. Cell proliferation was determined by Alamar blue assay (see Material and Methods). Growth rate is reported as relative values (means $\pm \mathrm{SE}$ ) to those of control cells cultured in growth medium alone. ${ }^{*} \mathrm{P}<0.05$, compared to control (ANOVA).

\section{Platinum drug sensitivity of ovarian cancer cells}

To further confirm the equitoxic dose, the sensitivity to the drugs was determined by clonogenic assay using SKOV-3 cells (Figure 2A). Consistent with the cell cytotoxicity observations, we found that equitoxic doses of these platinum drugs showed similar reductions in the number of colonies (Figure 2B). The drug-treated cells had less than $10 \%$ survival compared with untreated
A

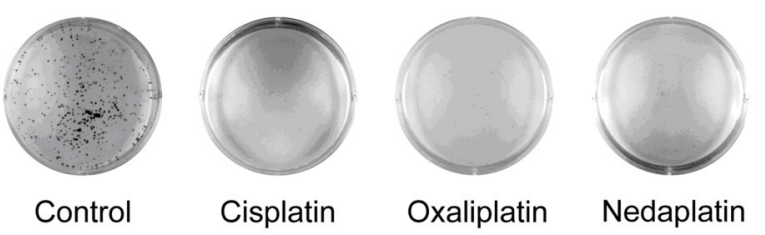

$\mathrm{B}$

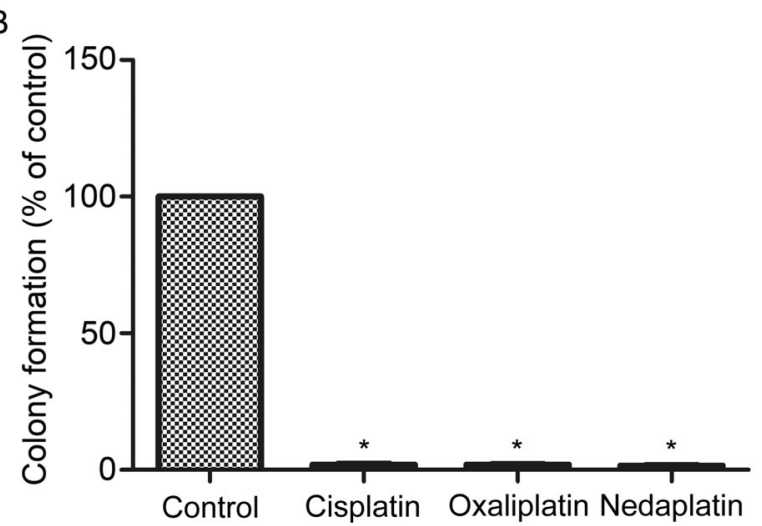

Figure 2. Effect of $1 \mu \mathrm{g} / \mathrm{mL}$ cisplatin, $8 \mu \mathrm{g} / \mathrm{mL}$ oxaliplatin, and $8 \mu \mathrm{g} / \mathrm{mL}$ nedaplatin on SKOV-3 cells in clonogenic assay. $A$, Photographs in a representative experiment are shown. $B$, The graph was calculated from the number of colonies formed on the culture plate 10 days after treatment. Clonogenic survival was expressed as surviving fractions for treatment with equitoxic dose of platinum drugs. Data are reported as percentage of colonies compared with untreated control (means $\pm S E$ ). ${ }^{*} \mathrm{P}<0.001$, compared to control (ANOVA).

control groups. We thus confirmed that equitoxic doses of the drugs had equal potency as measured by this assay of drug sensitivity.

Platinum drugs inhibit migration in ovarian cancer cells

To investigate the effects of platinum drugs on migration of ovarian cancer cells, an in vitro wound healing assay was performed (Figure $3 \mathrm{~A}$ ). As shown in Figure 3B, oxaliplatin and nedaplatin inhibited SKOV-3 cell migration by less than $10 \%$, and cisplatin suppressed it only slightly. This suggests that oxaliplatin and nedaplatin are more effective than cisplatin in inhibiting ovarian cancer cell migration.

\section{Effects of platinum chemotherapy on STAT3 signaling}

We used SKOV-3 cells as a representative cell line to determine the effects of platinum drugs on STAT3 signaling in ovarian cancer. SKOV-3 is a serous adenocarcinoma cell line representative of $\sim 80 \%$ of all ovarian cancers, and contains high levels of phosphorylated STAT3 $(16,17)$. Furthermore, SKOV-3 cells, originally obtained from a patient with intrinsic resistance to clinically achievable doses of cisplatin, show intrinsic resistance to cisplatin treatment, which could contribute to 
A

$\mathrm{Oh}$
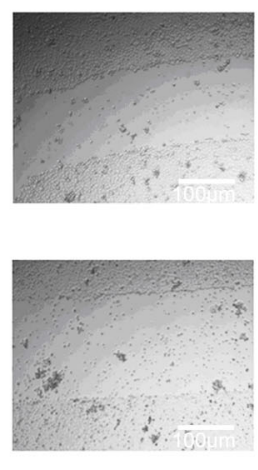

Cisplatin
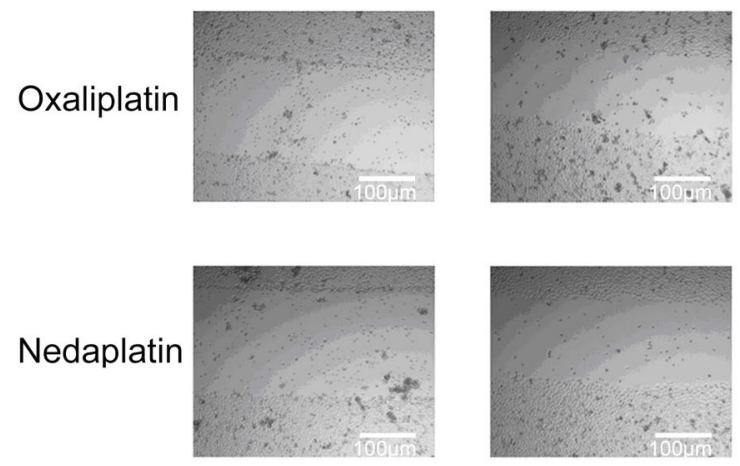

B

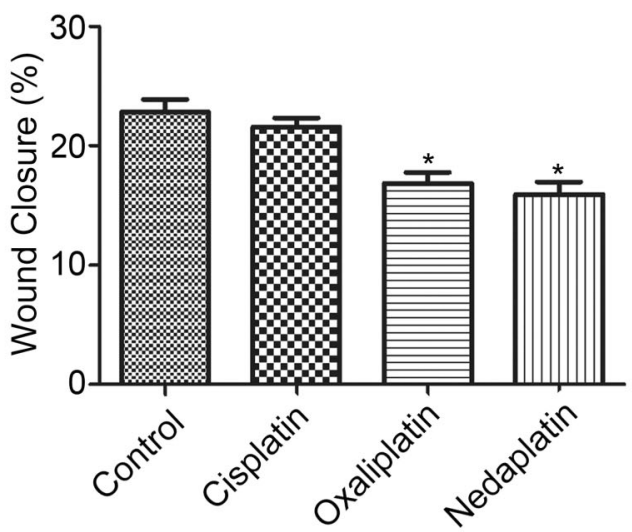

Figure 3. Effect of $1 \mu \mathrm{g} / \mathrm{mL}$ cisplatin, $8 \mu \mathrm{g} / \mathrm{mL}$ oxaliplatin, and $8 \mu \mathrm{g} / \mathrm{mL}$ nedaplatin on migration of SKOV-3 cells. Cell monolayers were scraped by a sterile micropipette tip and the cells were treated with equitoxic doses of platinum drugs for $24 \mathrm{~h}$. A, SKOV-3 cells migrated to the wounded region were photographed at 0 and $24 \mathrm{~h}$. $B$, The wound area of the cultures was quantified in three fields for each treatment. Data are reported as means $\pm \mathrm{SE}$. ${ }^{*} \mathrm{P}<0.01$, compared to control (ANOVA).

identifying clinically relevant resistance mechanisms (7). SKOV-3 cells were treated for $24 \mathrm{~h}$ with equitoxic doses of platinum drugs that had approximately $90 \%$ viability to examine their effects on STAT3 (Figure 1).
Cisplatin led to a marked induction of pSTAT3 (Tyr705) that leveled off at $3 \mathrm{~h}$ (Figure 4D). With oxaliplatin, pSTAT3 (Tyr705) decreased from $15 \mathrm{~min}$ to $1 \mathrm{~h}$ and then increased to a peak at $3 \mathrm{~h}$, and with nedaplatin it decreased from $15 \mathrm{~min}$ to $3 \mathrm{~h}$ and then increased to a peak at $6 \mathrm{~h}$. Cisplatin treatment was followed by a pSTAT3 (Ser727) peak at $6 \mathrm{~h}$ (Figure 4E). However, oxaliplatin resulted in an induction at $15 \mathrm{~min}$ and then a decrease in pSTAT3 (Ser727) (Figure 4E). Similar results were obtained with nedaplatin treatment (Figure 4E).

STAT3 $\alpha$ increased to a peak at $1 \mathrm{~h}$ and then decreased (Figure 4F), while STAT3 $\beta$ decreased to a minimum at $3 \mathrm{~h}$ and then increased from 3 to $24 \mathrm{~h}$ (Figure $4 \mathrm{G}$ ) after cisplatin treatment. Following oxaliplatin treatment, STAT3 $\alpha$ decreased from 15 min to $3 \mathrm{~h}$, followed by an increase at $6 \mathrm{~h}$, and then a decrease at $24 \mathrm{~h}$ (Figure $4 \mathrm{~F}$ ), whereas STAT3 $\beta$ increased by about $20 \%$ in this experiment from $15 \mathrm{~min}$ to $1 \mathrm{~h}$, followed by a reduction (Figure 4G). However, nedaplatin inhibited STAT3 $\alpha$ at $15 \mathrm{~min}$ and then slightly increased (Figure 4F), while it increased STAT3 $\beta$ as early as $15 \mathrm{~min}$ followed by a decrease (Figure 4G).

The data showed substantial decreases in both total STAT3 and phosphorylated STAT3 at $24 \mathrm{~h}$, suggesting that platinum drugs block the STAT3 pathway. However, oxaliplatin and nedaplatin had different effects on STAT3 signaling than cisplatin during the early time course.

\section{Effects of platinum chemotherapy on STAT3 target proteins}

To investigate the downstream consequences of STAT3 inhibition, Western blotting was performed to determine the protein levels of VEGF, cyclin D1, survivin, and $\mathrm{BCl}-\mathrm{X}_{\mathrm{L}}$ following indicated times of exposure to platinum drugs at an equitoxic dose. There was a clear induction in VEGF at $1 \mathrm{~h}$ post-cisplatin treatment, and a slight increase at $1 \mathrm{~h}$ post-oxaliplatin treatment, whereas nedaplatin slightly increased VEGF at $6 \mathrm{~h}$ (Figure 5D). Figure $5 \mathrm{E}$ shows that cyclin $\mathrm{D} 1$ levels were reduced at $15 \mathrm{~min}$ and then slightly increased after cisplatin treatment. With oxaliplatin, cyclin D1 levels decreased at first and then slightly increased at $3 \mathrm{~h}$. Nedaplatin showed a similar trend, except that it increased after $6 \mathrm{~h}$. We also observed significant elevation of survivin in cisplatintreated cells from $15 \mathrm{~min}$ to $1 \mathrm{~h}$, followed by a temporary decrease, and finally an induction at $24 \mathrm{~h}$ (Figure 5F). Oxaliplatin induced survivin at $15 \mathrm{~min}$, and then responses similar to those observed for cisplatin were seen. However, nedaplatin decreased survivin at $15 \mathrm{~min}$ and then slightly increased it. Cisplatin induced a small increase in $\mathrm{Bcl}-\mathrm{X}_{\mathrm{L}}$ at $15 \mathrm{~min}$, which then decreased (Figure 5G). Both oxaliplatin and nedaplatin at first decreased $B c l-X_{L}$ expression, but then increased it to a peak at $1 \mathrm{~h}$ with oxaliplatin and $6 \mathrm{~h}$ with nedaplatin. Although these platinum agents had similar effects on 


\section{A Cisplatin}

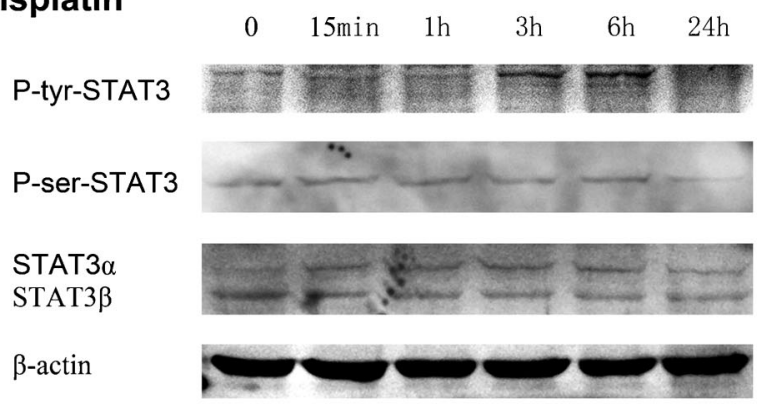

\section{B Oxaliplatin}

\section{$0 \quad 15 \mathrm{~min} \quad 1 \mathrm{~h} \quad 3 \mathrm{~h} \quad 6 \mathrm{~h} \quad 24 \mathrm{~h}$}

P-tyr-STAT3

P-ser-STAT3

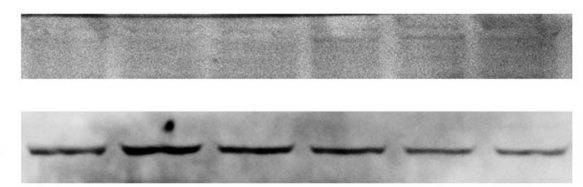

STAT $3 \alpha$

STAT3 $\beta$

$\beta$-actin

\section{Nedaplatin}

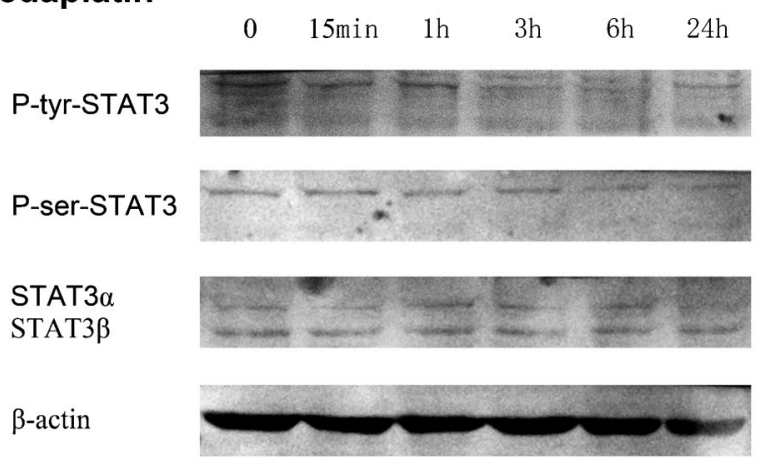

D

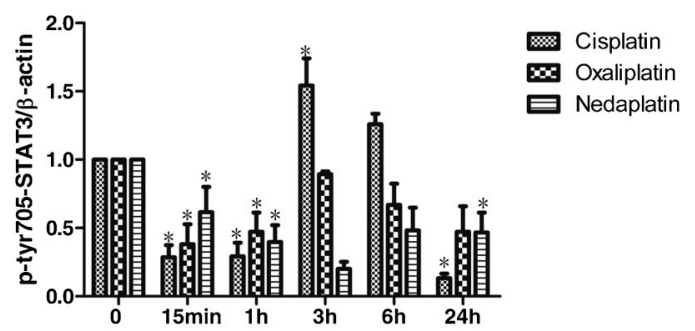

$\mathbf{E}$

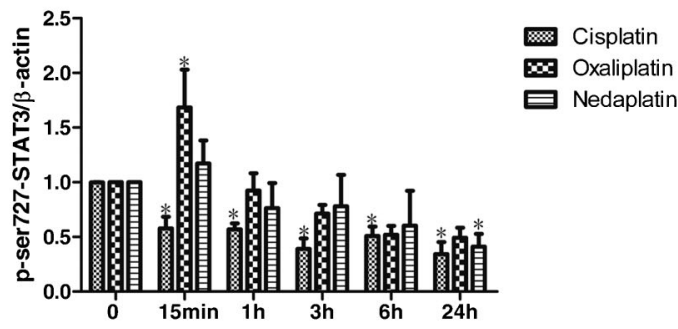

$\mathbf{F}$

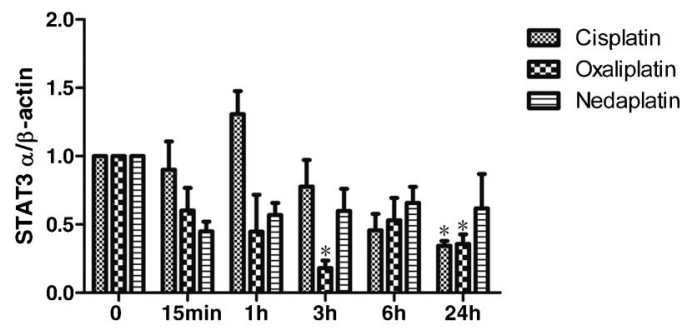

$\mathbf{G}$

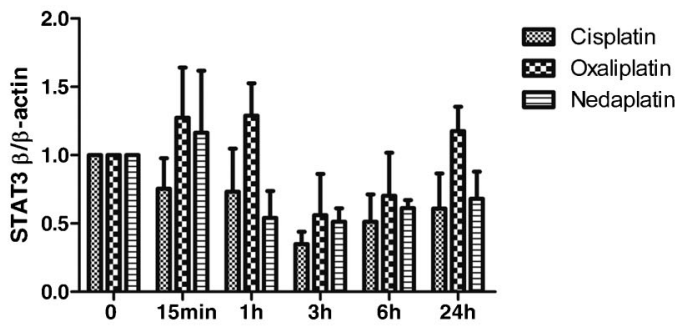

Figure 4. Effect of platinum drugs on STAT3 and phosphorylation of STAT3. SKOV-3 cells were treated with equitoxic doses of $1 \mu \mathrm{g} /$ $\mathrm{mL}$ cisplatin $(A), 8 \mu \mathrm{g} / \mathrm{mL}$ oxaliplatin $(B)$, and $8 \mu \mathrm{g} / \mathrm{mL}$ nedaplatin $(C)$ for indicated times. Phosphorylated and total STAT3 was analyzed by immunoblot. $\beta$-actin was used as a loading control. The density of the bands was quantitated, and the ratio of antibodies to $\beta$-actin is shown. The relative density was divided by the control. A, B, C, Immunoblot images of STAT3 and phosphorylation of STAT3 in platinum-treated SKOV-3 cells. D, E, F, G, Quantitative results of STAT3 and phosphorylation of STAT3 bands are shown. Data are reported as percent of control (means $\pm \mathrm{SE}$ ). ${ }^{*} \mathrm{P}<0.05$, compared to control (ANOVA).

STAT3 targeted proteins at $24 \mathrm{~h}$, their early effects differed. The overall results indicated that the STAT3 pathway correlated with the inhibition of growth of SKOV-3 cells by these three platinum drugs.

\section{Discussion}

Although STAT3-targeted therapies could potentiate the effect of cisplatin $(8,12)$, some researchers found that pretreatment of platinum-resistant cells with AG490 (a specific STAT3 inhibitor) resulted in no significant increase in sensitivity to cisplatin (18). The different effects of targeting STAT3 may be caused by the varying threshold activation of STAT3 depending on the endogenous level of phosphorylated STAT3 of different cancer cell types (13). Thus, we first investigated effects on STAT3 of platinum drugs in ovarian cancer to confirm whether they could affect STAT3 signaling. Activation of STAT3, an adverse prognostic factor, is associated with cisplatin resistance in epithelial malignancies $(1,7,8)$, 


\section{A Cisplatin}

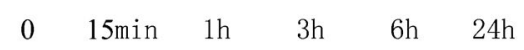

VEGF

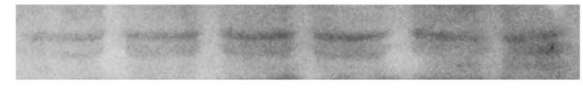

Cyclin D1

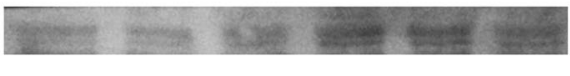

Survivin

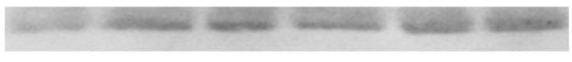

$\mathrm{Bcl}-\mathrm{XL}$

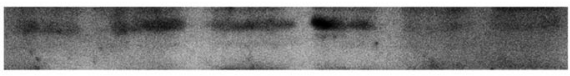

$\beta$-actin

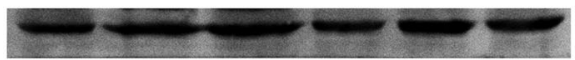

\section{B Oxaliplatin}
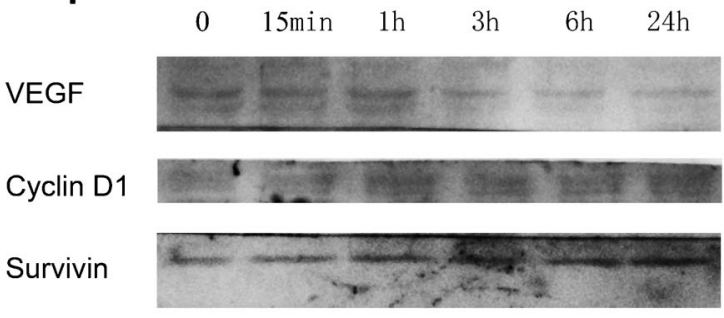

$\mathrm{Bcl-XL}$

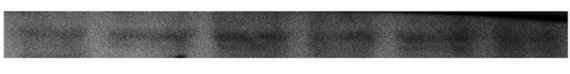

$\beta$-actin

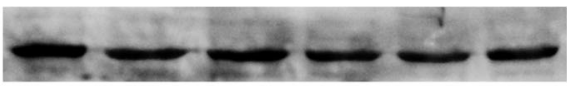

\section{Nedaplatin}

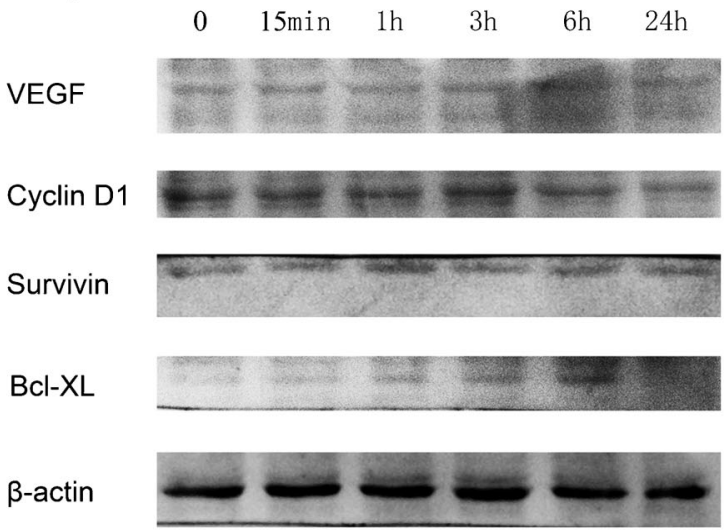

D

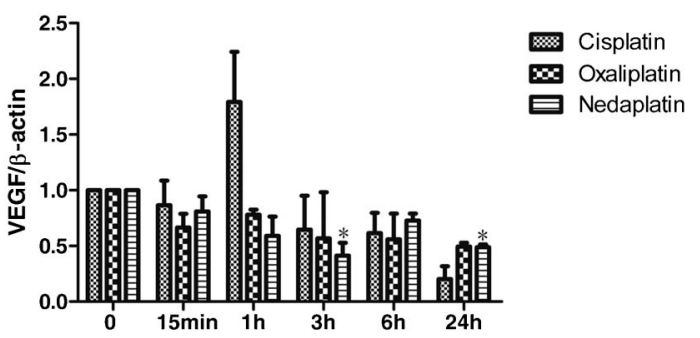

E

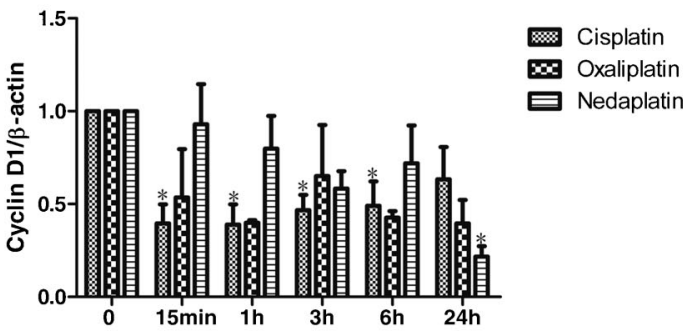

F

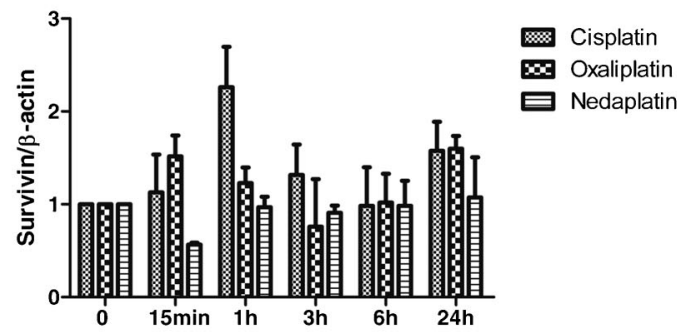

G

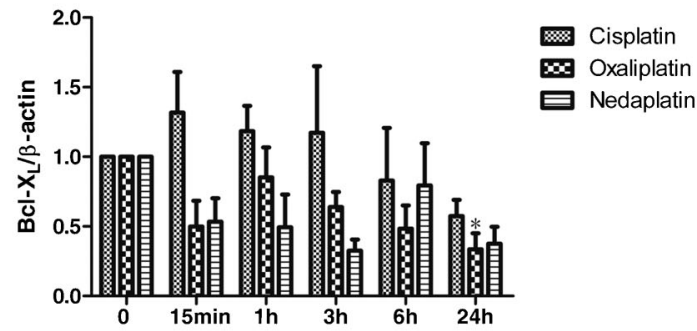

Figure 5. Effect of platinum drugs on downstream signaling of STAT3. SKOV-3 cells were treated with equitoxic doses of $1 \mu \mathrm{g} / \mathrm{mL}$ cisplatin $(A), 8 \mu \mathrm{g} / \mathrm{mL}$ oxaliplatin $(B), 8 \mu \mathrm{g} / \mathrm{mL}$ nedaplatin $(C)$ for indicated times and analyzed by immunoblot for the indicated antibodies. $\beta$-actin was used as an internal control. $A, B, C$, Immunoblot images of VEGF, cyclin D1, survivin, and Bcl- $\mathrm{X}_{\mathrm{L}}$ in platinumtreated SKOV-3 cells. $D, E, F, G$, Quantitative results of VEGF, cyclin D1, survivin, and Bcl- $\mathrm{X}_{\mathrm{L}}$ bands are shown. The density of the bands was quantitated, and normalized to $\beta$-actin as a loading control. The relative density is reported as percent of control (means $\pm \mathrm{SE}$ ). ${ }^{*} \mathrm{P}<0.05$, compared to control (ANOVA).

while cisplatin resistance positively correlates with colonyforming ability (11). However, we observed that equitoxic doses of platinum drugs had similar inhibitory effects on the STAT3 pathway at $24 \mathrm{~h}$ and on colony-forming ability in SKOV-3 cells, which is consistent with the findings that the ability to inhibit STAT3 signaling positively correlates 
with the cytotoxic effects of chemotherapy agents (19).

Because initial exposure to a chemotherapy drug may induce low, but clinically significant transient resistance, some early events might contribute to the onset of acquired chemoresistance in ovarian cancer (20). The rapid acquisition of resistance may represent physiological adaptation at the multicellular level, which could render normal and cancerous epithelial cells less vulnerable to drug-induced apoptosis (20-22). Thus, a relatively larger percentage of viable tumor cells would remain after chemotherapy (23) and the surviving tumor cells reprogrammed by drugs might induce profound drug-resistance effects. It has been reported that changes in relevant drug-resistance-associated gene expression promote reduction of the initial effectiveness of chemotherapy drugs in ovarian cancer (20). The induction of genes related to DNA replication and repair in cisplatin-treated ovarian cancer are associated with immediate compensatory responses to treatment (20). STAT3 correlates with cisplatin resistance and DNA repair by preventing telomere shortening (24). Therefore, we concluded that the early response of STAT3 induced by platinum agents might be correlated with cisplatin resistance in ovarian cancer. To confirm our hypothesis, we investigated early responses of the STAT3 pathway to platinum drugs and found that cisplatin induced pSTAT3 (Tyr705), STAT3 $\alpha$, VEGF, survivin, and $\mathrm{BCl}-\mathrm{X}_{\mathrm{L}}$ for short periods, which promotes ovarian cancer progression, while oxaliplatin and nedaplatin temporarily induced pSTAT3 (Ser727) and STAT3 $\beta$, which may inhibit STAT3 activity and reverse cisplatin resistance.

We demonstrated that the inhibitory effects of equitoxic platinum drugs on migration of SKOV-3 cells are different (Figure 2). Ovarian cancers metastasize through shedding cells into the peritoneal cavity (13), which associate with migration. Moreover, tyrosine phosphorylated STAT3 may be located at the leading edge of migrating cells, thus promoting migration (9). Therefore, we concluded that induction of pSTAT3 (Tyr705) cisplatin in the early time course could contribute to the migration of ovarian cancer cells, which is probably not associated with its toxicity.

Phosphorylation at tyrosine 705 resulting in dimerization, activation, and translocation of STAT3 is mainly associated with the oncogenic status of STAT3 (12). However, STAT3 serine phosphorylation in response to DNA damage may inhibit the tyrosine phosphorylation of STAT3 $(12,25,26)$. Given our findings, we speculated that oxaliplatin and nedaplatin may inactivate STAT3 through upregulation of pSTAT3 (Ser727), concurrent with downregulation of pSTAT3 (Tyr705) in ovarian cancer cells at an early time, which may be correlated with reversal of cisplatin resistance.

Recent reports mainly focus on new agents that target STAT3 tyrosyl phosphorylation instead of total STAT3, and which inhibit tumor growth, promote apoptosis and reverse cisplatin resistance in ovarian cancer cells
$(7,8,25,27)$. However, unphosphorylated STAT3 is also important for oncogenesis and can affect gene expression, translocation to the nucleus, and binding to focal adhesions, thus affecting many forms of STAT3 signaling $(7,9,19)$. Total STAT3 includes STAT3 $\alpha$ and STAT3 $\beta$. STAT3 $\beta$ functions as a dominant negative when overexpressed in cells (26). However, STAT3 $\beta$ lacks the serine phosphorylation site within the carboxy-terminal transcriptional activation domain (26). STAT3 serine phosphorylation may enhance STAT3-mediated transcription $(12,25,26)$. It is possible that STAT3 $\beta$ and pSTAT3 (Ser727) inhibit STAT3 transcription in different ways. Temporary induction of STAT3 $\beta$ and pSTAT3 (Ser727) by oxaliplatin and nedaplatin might contribute by inhibiting transcription of STAT3.

High-grade ovarian cancers are characterized by the STAT3-induced gene expression associated with the development of chemoresistance, including activation of antiapoptotic proteins such as $\mathrm{Bcl}-\mathrm{X}_{\mathrm{L}}$ and survivin, upregulating cell cycle regulators (cyclin D1/D2), inducing angiogenic factors such as VEGF, repressing the transcription of p53 and nitric oxide synthase, and downregulating negative regulators such as necdin $(8,9,28)$. The agents targeting STAT3 could also downregulate the STAT3 target oncogenes that are mentioned above $(25,27,29-31)$. Since platinum drugs affect STAT3, we determined their effects downstream of STAT3, which may contribute to cisplatin resistance.

The activation of STAT3 induces a prolonged increase in the angiogenic protein VEGF, which plays a key role in embryonic angiogenesis, inducing invasiveness, tumor growth and metastasis $(32,33)$. The onset of angiogenesis is believed to be an early event in tumorigenesis and may facilitate tumor progression and metastasis. Diindolylmethane enhances cisplatin's ability to inhibit both VEGF expression and VEGF secretion through STAT3 (12). STAT3 may participate in the signal transduction pathways activated by VEGF in the interaction of ovarian carcinoma cells and progenitor cells (34). We discovered that cisplatin induced VEGF at $1 \mathrm{~h}$, which was paralleled by effects on STAT3 $\alpha$, while oxaliplatin and nedaplatin decreased it. Cisplatin might induce VEGF through induction of STAT $\alpha \alpha$ at an early stage, thus promoting angiogenesis of ovarian cancer.

Cyclin D1 is one of the G1 cyclins, controlling the proliferation of eukaryotic cells at the $\mathrm{G} 1$ to $\mathrm{S}$ transition. The trend of increasing cyclin D1 after cisplatin treatment was similar to induction of pSTAT3 (Tyr705), while oxaliplatin and nedaplatin showed the opposite trend, which supports previous studies that inhibition of STAT3 leads to obvious reduction in expression of cyclin D1 (35). Cisplatin might induce cyclin D1 through induction of pSTAT3 (Tyr705) at an early stage, and thus promote ovarian cancer cell proliferation.

Survivin is expressed during the G2/M phase of the cell cycle and inhibits the initiation of the intrinsic 
mitochondrial pathway of apoptosis (36), which is associated with resistance to cisplatin-induced apoptosis (37). In esophageal cancer, oxaliplatin-induced apoptosis could be partially caused by the early downregulation of survivin (38). However, our experiment showed that platinum drugs induced survivin expression at $24 \mathrm{~h}$, which supports previous reports that cisplatin drastically upregulated survivin in SKOV-3 cells (12). Targeting survival pathways could partially overcome chemotherapy resistance in resistant cells (39). Since it has been reported that STAT3 signaling is not involved in upregulation of survivin $(36,38)$ and activation of pSTAT3 correlates with proliferation but not with apoptosis (10), survivin might be partially associated with cisplatin resistance and may be affected mainly by upstream pathways other than the STAT3 pathway.

Inhibition of $\mathrm{Bcl}-\mathrm{X}_{\mathrm{L}}$ by agents targeting STAT3 increases the activity of chemotherapeutic drugs and contributes to the induction of apoptosis $(8,35)$. Bcl- $X_{L}$, which is regulated by both STAT3 (Tyr705) and (Ser727), blocks cytochrome $\mathrm{C}$ release in response to apoptotic stimuli, and correlates with chemoresistance in ovarian cancer $(8,35,36)$. However, effects of oxaliplatin on Bcl- $X_{L}$ depend on the cancer cell type (38). We found that $B c l-X_{L}$ was increased by cisplatin during the early period, but was

\section{References}

1. Cannistra SA. Cancer of the ovary. N Engl J Med 2004; 351: 2519-2529, doi: 10.1056/NEJMra041842.

2. Bristow RE, Chi DS. Platinum-based neoadjuvant chemotherapy and interval surgical cytoreduction for advanced ovarian cancer: a meta-analysis. Gynecol Oncol 2006; 103: 1070-1076, doi: 10.1016/j.ygyno.2006.06.025.

3. Koshiyama M, Kinezaki M, Uchida T, Sumitomo M. Chemosensitivity testing of a novel platinum analog, nedaplatin (254-S), in human gynecological carcinomas: a comparison with cisplatin. Anticancer Res 2005; 25: 4499-4502.

4. Chollet $\mathrm{P}$, Bensmaine MA, Brienza S, Deloche $\mathrm{C}$, Cure $\mathrm{H}$, Caillet $\mathrm{H}$, et al. Single agent activity of oxaliplatin in heavily pretreated advanced epithelial ovarian cancer. Ann Oncol 1996; 7: 1065-1070, doi: 10.1093/oxfordjournals.annonc. a010500.

5. Ferrandina G, Ludovisi M, De Vincenzo R, Salutari V, Lorusso $\mathrm{D}$, Colangelo $\mathrm{M}$, et al. Docetaxel and oxaliplatin in the second-line treatment of platinum-sensitive recurrent ovarian cancer: a phase II study. Ann Oncol 2007; 18: 13481353, doi: 10.1093/annonc/mdm136.

6. Wheate NJ, Walker S, Craig GE, Oun R. The status of platinum anticancer drugs in the clinic and in clinical trials. Dalton Trans 2010; 39: 8113-8127, doi: 10.1039/c0dt00292e.

7. Han Z, Hong Z, Gao Q, Chen C, Hao Z, Ji T, et al. A potent oncolytic adenovirus selectively blocks the STAT3 signaling pathway and potentiates cisplatin antitumor activity in ovarian cancer. Hum Gene Ther 2012; 23: 32-45, doi: 10.1089/hum.2011.101.

8. Duan Z, Foster R, Bell DA, Mahoney J, Wolak K, Vaidya A, decreased by oxaliplatin and nedaplatin. Thus, early induction of $\mathrm{Bcl}-\mathrm{X}_{\mathrm{L}}$ may be associated with the development of cisplatin resistance in ovarian cancer.

Platinum agents have similar effects on the STAT3 pathway at $24 \mathrm{~h}$, which are associated with drug cytotoxicity. Since initial exposure to a drug may induce transient resistance, early induction of pSTAT3 (Tyr705), STAT3 $\alpha$, VEGF, survivin, and $B c l-X_{L}$ after cisplatin treatment, and reduction of pSTAT3 (Ser727) and STAT3 $\beta$ might promote cancer progression and lead to cancer relapse. Thus, oxaliplatin and nedaplatin show opposite effects in comparison with cisplatin, which may explain their reversal of cisplatin resistance. This is the first report showing that early responses of the STAT3 pathway to platinum drugs were associated with cisplatin resistance in epithelial ovarian cancer. This study underscores the potential importance of the drug resistance mechanisms induced by early cellular responses to initial drug exposure and provides a rationale for new therapeutic strategies in reversing cisplatin resistance.

\section{Acknowledgments}

Research supported by the National Natural Science Foundation of China (\#30973188).

et al. Signal transducers and activators of transcription 3 pathway activation in drug-resistant ovarian cancer. Clin Cancer Res 2006; 12: 5055-5063, doi: 10.1158/1078-0432. CCR-06-0861.

9. Silver DL, Naora H, Liu J, Cheng W, Montell DJ. Activated signal transducer and activator of transcription (STAT) 3 : localization in focal adhesions and function in ovarian cancer cell motility. Cancer Res 2004; 64: 3550-3558, doi: 10.1158/0008-5472.CAN-03-3959.

10. Rosen DG, Mercado-Uribe I, Yang G, Bast RC Jr, Amin HM, Lai $R$, et al. The role of constitutively active signal transducer and activator of transcription 3 in ovarian tumorigenesis and prognosis. Cancer 2006; 107: 27302740, doi: 10.1002/cncr.22293.

11. Yue $P$, Zhang $X$, Paladino D, Sengupta B, Ahmad S, Holloway RW, et al. Hyperactive EGF receptor, Jaks and Stat3 signaling promote enhanced colony-forming ability, motility and migration of cisplatin-resistant ovarian cancer cells. Oncogene 2012; 31: 2309-2322, doi: 10.1038/onc. 2011.409.

12. Kandala PK, Srivastava SK. Diindolylmethane suppresses ovarian cancer growth and potentiates the effect of cisplatin in tumor mouse model by targeting signal transducer and activator of transcription 3 (STAT3). BMC Med 2012; 10: 9, doi: 10.1186/1741-7015-10-9.

13. Colomiere M, Findlay J, Ackland L, Ahmed N. Epidermal growth factor-induced ovarian carcinoma cell migration is associated with JAK2/STAT3 signals and changes in the abundance and localization of alpha6beta1 integrin. Int $J$ Biochem Cell Biol 2009; 41: 1034-1045, doi: 10.1016/ 
j.biocel.2008.09.018.

14. Hall J, Guo G, Wray J, Eyres I, Nichols J, Grotewold L, et al. Oct4 and LIF/Stat3 additively induce Kruppel factors to sustain embryonic stem cell self-renewal. Cell Stem Cell 2009; 5: 597-609, doi: 10.1016/j.stem.2009.11.003.

15. Takaishi K, Komohara Y, Tashiro H, Ohtake H, Nakagawa $\mathrm{T}$, Katabuchi $\mathrm{H}$, et al. Involvement of M2-polarized macrophages in the ascites from advanced epithelial ovarian carcinoma in tumor progression via Stat3 activation. Cancer Sci 2010; 101: 2128-2136, doi: 10.1111/j.1349-7006.2010. 01652.x.

16. Meinhold-Heerlein I, Bauerschlag D, Hilpert F, Dimitrov P, Sapinoso LM, Orlowska-Volk M, et al. Molecular and prognostic distinction between serous ovarian carcinomas of varying grade and malignant potential. Oncogene 2005; 24: 1053-1065, doi: 10.1038/sj.onc. 1208298.

17. Gest C, Mirshahi P, Li H, Pritchard LL, Joimel U, Blot E, et al. Ovarian cancer: Stat3, RhoA and IGF-IR as therapeutic targets. Cancer Lett 2012; 317: 207-217, doi: 10.1016/ j.canlet.2011.11.026.

18. Roberts D, Schick J, Conway S, Biade S, Laub PB, Stevenson JP, et al. Identification of genes associated with platinum drug sensitivity and resistance in human ovarian cancer cells. $\mathrm{Br} J$ Cancer 2005; 92: 1149-1158, doi: 10.1038/sj.bjc.6602447.

19. Walker SR, Chaudhury M, Nelson EA, Frank DA. Microtubule-targeted chemotherapeutic agents inhibit signal transducer and activator of transcription 3 (STAT3) signaling. Mol Pharmacol 2010; 78: 903-908, doi: 10.1124/ mol.110.066316.

20. L'Esperance S, Bachvarova M, Tetu B, Mes-Masson AM, Bachvarov D. Global gene expression analysis of early response to chemotherapy treatment in ovarian cancer spheroids. BMC Genomics 2008; 9: 99, doi: 10.1186/14712164-9-99.

21. Bates RC, Buret A, van Helden DF, Horton MA, Burns GF. Apoptosis induced by inhibition of intercellular contact. $J$ Cell Biol 1994; 125: 403-415, doi: 10.1083/jcb.125.2.403.

22. Frisch $\mathrm{SM}$, Francis $\mathrm{H}$. Disruption of epithelial cell-matrix interactions induces apoptosis. J Cell Biol 1994; 124: 619626, doi: 10.1083/jcb.124.4.619.

23. Blazer DG III, Kishi Y, Maru DM, Kopetz S, Chun YS, Overman MJ, et al. Pathologic response to preoperative chemotherapy: a new outcome end point after resection of hepatic colorectal metastases. J Clin Oncol 2008; 26: 53445351, doi: 10.1200/JCO.2008.17.5299.

24. Konnikova L, Simeone MC, Kruger MM, Kotecki M, Cochran $\mathrm{BH}$. Signal transducer and activator of transcription 3 (STAT3) regulates human telomerase reverse transcriptase (hTERT) expression in human cancer and primary cells. Cancer Res 2005; 65: 6516-6520, doi: 10.1158/00085472.CAN-05-0924.

25. Selvendiran K, Tong L, Bratasz A, Kuppusamy ML, Ahmed $\mathrm{S}$, Ravi $\mathrm{Y}$, et al. Anticancer efficacy of a difluorodiarylidenyl piperidone (HO-3867) in human ovarian cancer cells and tumor xenografts. Mol Cancer Ther 2010; 9: 1169-1179, doi: 10.1158/1535-7163.MCT-09-1207.

26. O'Shea JJ, Gadina M, Schreiber RD. Cytokine signaling in 2002: new surprises in the Jak/Stat pathway. Cell 2002; 109
(Suppl): S121-S131, doi: 10.1016/S0092-8674(02)00701-8.

27. Selvendiran K, Ahmed S, Dayton A, Kuppusamy ML, Rivera BK, Kalai T, et al. HO-3867, a curcumin analog, sensitizes cisplatin-resistant ovarian carcinoma, leading to therapeutic synergy through STAT3 inhibition. Cancer Biol Ther 2011; 12: 837-845, doi: 10.4161/cbt.12.9.17713.

28. Haviland R, Eschrich S, Bloom G, Ma Y, Minton S, Jove R, et al. Necdin, a negative growth regulator, is a novel STAT3 target gene down-regulated in human cancer. PLOS One 2011; 6: e24923, doi: 10.1371/journal.pone.0024923.

29. Huang S, Chen M, Shen Y, Shen W, Guo H, Gao Q, et al. Inhibition of activated Stat3 reverses drug resistance to chemotherapeutic agents in gastric cancer cells. Cancer Lett 2012; 315: 198-205, doi: 10.1016/j.canlet.2011.10.011.

30. Guo Y, Nemeth J, O'Brien C, Susa M, Liu X, Zhang Z, et al. Effects of siltuximab on the IL-6-induced signaling pathway in ovarian cancer. Clin Cancer Res 2010; 16: 5759-5769, doi: 10.1158/1078-0432.CCR-10-1095.

31. Selvendiran K, Kuppusamy ML, Ahmed S, Bratasz A Meenakshisundaram G, Rivera BK, et al. Oxygenation inhibits ovarian tumor growth by downregulating STAT3 and cyclin-D1 expressions. Cancer Biol Ther 2010; 10: 386-390, doi: 10.4161/cbt.10.4.12448.

32. Carmeliet P, Jain RK. Angiogenesis in cancer and other diseases. Nature 2000; 407: 249-257, doi: 10.1038/ 35025220

33. Wang FQ, So J, Reierstad S, Fishman DA. Vascular endothelial growth factor-regulated ovarian cancer invasion and migration involves expression and activation of matrix metalloproteinases. Int J Cancer 2006; 118: 879-888, doi: 10.1002/ijc.21421.

34. Chen H, Ye D, Xie X, Chen B, Lu W. VEGF, VEGFRs expressions and activated STATs in ovarian epithelial carcinoma. Gynecol Oncol 2004; 94: 630-635, doi: 10.1016/j.ygyno.2004.05.056.

35. Lee JH, Schutte D, Wulf G, Fuzesi L, Radzun HJ, Schweyer $\mathrm{S}$, et al. Stem-cell protein Piwil2 is widely expressed in tumors and inhibits apoptosis through activation of Stat3/ Bcl-XL pathway. Hum Mol Genet 2006; 15: 201-211, doi: 10.1093/hmg/ddi430.

36. Siddiqa A, Long LM, Li L, Marciniak RA, Kazhdan I. Expression of HER-2 in MCF-7 breast cancer cells modulates anti-apoptotic proteins Survivin and $\mathrm{Bcl}-2$ via the extracellular signal-related kinase (ERK) and phosphoinositide-3 kinase (PI3K) signalling pathways. BMC Cancer 2008; 8: 129, doi: 10.1186/1471-2407-8-129.

37. Asechi H, Hatano E, Nitta T, Tada M, Iwaisako K, Tamaki N et al. Resistance to cisplatin-induced apoptosis via PI3Kdependent survivin expression in a rat hepatoma cell line. Int J Oncol 2010; 37: 89-96.

38. Ngan CY, Yamamoto H, Takagi A, Fujie Y, Takemasa I, Ikeda $\mathrm{M}$, et al. Oxaliplatin induces mitotic catastrophe and apoptosis in esophageal cancer cells. Cancer Sci 2008; 99: 129-139.

39. Bose D, Zimmerman LJ, Pierobon M, Petricoin E, Tozzi F, Parikh A, et al. Chemoresistant colorectal cancer cells and cancer stem cells mediate growth and survival of bystander cells. Br J Cancer 2011; 105: 1759-1767, doi: 10.1038/ bjc.2011.449. 